\title{
Erratum to: Co-culture of Retinal and Endothelial Cells Results in the Modulation of Genes Critical to Retinal Neovascularization
}

\author{
Vascular Cell 4:6 ～DOI: 10.1186/2045-824X-4-6 ～～c Li et al.; licensee Publiverse Online S.R.L. 2012 \\ Received: 14 Mar 2012 | Accepted: 26 Mar 2012 | Published: 26 Mar 2012 \\ Kumar Ravindra ${ }^{@}$, Harris-Hooker Sandra, Kumar Ritesh, Sanford Gary \\ + Contributed equally ${ }^{@}$ Corresponding author
}

\section{Correction}

Following publication of our article [ 1] it was noted that Figures five E and five G were the same as Figures six $\mathrm{A}$ and six B. Figure 1 in this correction article is the correct version of Figure six that should have been included in the original article [ 1]. We apologize for any inconvenience caused by this error. Figure 1

RT-PCR and Western blot analysis of NFxB. Total RNA and total protein were extracted from HRPC and HUVEC cultured alone or co-cultured under normoxia and hypoxia conditioned for $24 \mathrm{~h}$. The expression of NFxB was measured by (A) electrophoresis of RT-PCR, (C) Western blot analysis in the HRPC and HUVEC. Figures (B, D) the band intensities corresponding to the NFxB were quantified by image analysis using a BioRad scanning densitometer and Quantity One analysis software. Data were shown as ratio of NF $\mathcal{B}$ densities to that of 18S RNA for RT-PCR and $\beta$-actin antibody was used to normalize Western blot for differences in loading and the transfer efficiencies. All data were expressed as mean $+/-\mathrm{SE}$ and results are representatives of three independent experiments.

\section{Declarations}

\section{Authors' original submitted files for images}

Below are the links to the authors' original submitted files for images.

Authors' original file for figure 1

The online version of the original article can be found at 10.1186/2045-824X-3-27

\section{References}

1. Kumar R Harris-Hooker S Kumar R Sanford G Co-culture of Retinal and Endothelial Cells Results in the Modulation of Genes Critical to Retinal Neovascularization Vascular Cell 20113 273253041 10.1186/2045-824X-3-27 